\title{
Large-uncertainty intelligent states for angular momentum and angle
}

\author{
Jörg B Götte, Roberta Zambrini, Sonja Franke-Arnold and \\ Stephen M Barnett \\ Department of Physics, University of Strathclyde, Glasgow G4 0NG, UK \\ E-mail: joerg@phys.strath.ac.uk
}

\begin{abstract}
The equality in the uncertainty principle for linear momentum and position is obtained for states which also minimize the uncertainty product. However, in the uncertainty relation for angular momentum and angular position both sides of the inequality are state dependent and therefore the intelligent states, which satisfy the equality, do not necessarily give a minimum for the uncertainty product. In this paper, we highlight the difference between intelligent states and minimum uncertainty states by investigating a class of intelligent states which obey the equality in the angular uncertainty relation while having an arbitrarily large uncertainty product. To develop an understanding for the uncertainties of angle and angular momentum for the large-uncertainty intelligent states we compare exact solutions with analytical approximations in two limiting cases.
\end{abstract}

Submitted to: J. Opt. B: Quantum Semiclass. Opt.

PACS numbers: 03.65.Ta 40.25-p 


\section{Introduction}

The uncertainty principle limits the precise knowledge of all physical quantities of a system. For linear position and linear momentum this limit is expressed as a constant lower bound for the product of the respective uncertainties [1]:

$$
\Delta x \Delta p_{x} \geq \frac{\hbar}{2}
$$

Of a particular interest are states which minimize the uncertainty product, as they describe the quantum system as precisely as possible. In the linear case these states coincide with the intelligent states, that is states, which satisfy the equality in the uncertainty relation [2]. In the general case, where the bound in the uncertainty relation is state dependent, one has to distinguish between the intelligent states, states which minimize the uncertainty product under an additional constraint and states which give a global minimum $[3,4]$.

For angular variables like phase and rotation angle it is necessary to restrict the range of allowed angles to a $2 \pi$ radian interval and to define our observable within this range [5]. Naturally, the angular uncertainty, the uncertainty relation and the associated intelligent states will depend on our choice of the $2 \pi$ radian range. Changing the range of angles can greatly modify the angular uncertainty associated with any given state [6]. Here, we choose our window of angles to run from $-\pi$ to $\pi$. The uncertainty relation for orbital angular momentum and angular position has a state dependent lower bound $[7]$ :

$$
\Delta \phi \Delta L_{z} \geq \frac{\hbar}{2}|1-2 \pi P(\pi)|
$$

as it contains the angle probability density $P(\pi)$ at the angle $\pi$, corresponding to the end of our range of angles. For a general interval $\left[\theta_{0}, \theta_{0}+2 \pi\right)$, the probability density in the uncertainty relation (2) would be evaluated at the angle $\theta_{0}$ or $\theta_{0}+2 \pi$, as physical properties are periodic in the angle.

The form of the uncertainty relation (2) has been confirmed experimentally using light beams carrying orbital angular momentum [8]. It is well established that the spin angular momentum of a photon corresponds to the polarisation of a light beam and can be found with one of two values $\pm \hbar$ per photon for left- and right-handed circular polarisation. The orbital angular momentum, however, is associated with the azimuthal helical phase fronts $\exp (\mathrm{i} m \phi)$ and has values of $m \hbar$ per photon, where $m$ can take any integer value [9]. In the experimental verification of the uncertainty relation (2) a light beam with zero orbital angular momentum was passed through an angular aperture, which restricts the angular position and changes the angle probability density for the passing light beam [8]. The form of the aperture corresponded to the angle probability density of the intelligent states. According to the Heisenberg uncertainty principle a restriction of the angular position leads to a spread in the angular momentum distribution, which can be measured with various experimental techniques [10]. The 
intelligent states used in the confirmation of the uncertainty relation had the form of truncated Gaussians in the angle representation [8]

$$
\psi(\phi)=\frac{1}{N} \exp \left(-\frac{\lambda}{2} \phi^{2}\right), \quad-\pi \leq \phi<\pi \quad \lambda>0 .
$$

This class of states is characterised by a parameter $\lambda$ and for $\lambda>0$ the angle wavefunction of the intelligent states has a single peak at $\phi=0$, which is in the middle of the chosen $2 \pi$ radian interval. These states and their relation to the constrained minimum uncertainty states have been explored at some length $[3,8]$.

In this paper we focus on intelligent states with $\lambda<0$. In contrast to the intelligent states for $\lambda>0$, whose uncertainty product has an upper bound of $\hbar / 2$, intelligent states with $\lambda<0$ may have an arbitrarily large uncertainty product and yet still fulfil the equality in the uncertainty relation (2) [11]. This is because for $\lambda<0$ the angle wavefunction grows exponentially towards the boundaries of the interval $[-\pi, \pi)$ thus increasing the right hand side of the uncertainty relation.

In the following we will present an analysis of the large-uncertainty intelligent states, their angular momentum distribution and uncertainty product. Along with the analytically exact form of the intelligent states, we are able to describe the limiting behaviour of these states by means of some simple analytical approximations. This paper complements the analysis of the angular uncertainty relation started in $[3,8]$ and emphasizes the difference between intelligent and constrained minimum uncertainty product states in the angular case. These are states which minimize the uncertainty product for the constraint of a given uncertainty in either the angle or the angular momentum.

\section{The uncertainty relation}

The simplicity of the uncertainty relation (2) hides a number of mathematical subtleties. We can apply it with confidence to any physically preparable state but its derivation from angle and angular momentum observables requires the careful application of a limiting procedure [7] and we should treat (2) as an approximation, albeit an excellent one, to a more rigorous result. Careless manipulation can lead to difficulties and these have been ascribed to problems with self-adjointness of the observables [12]. It is clear, however, that such difficulties originate in the inappropriate application of (2). In this section we review the formulation of the angle operator and the derivation of the rigorous uncertainty relation. This will allow us to determine precisely the limits of validity of (2) and of the angle representation of the state as used in this paper [3].

We introduce the angle operator by working with a $(2 L+1)$-dimensional state space spanned by the eigenvectors $|m\rangle$ of the angular momentum operator, $\hat{L}_{z}$, with $m=-L,-L+1, \ldots,-1,0,1, \ldots, L$. At a later stage, and only after physical results have been calculated, we shall allow $L$ to tend to infinity. Within the state space we 
can construct a complete set of orthonormal angle eigenstates of the form

$$
\left|\theta_{n}\right\rangle=(2 L+1)^{-1 / 2} \sum_{m=-L}^{L} \exp \left(-\mathrm{i} m \theta_{n}\right)|m\rangle,
$$

where

$$
\theta_{n}=\theta_{0}+\frac{2 \pi n}{2 L+1}, \quad(n=0,1, \ldots, 2 L) .
$$

The choice of $\theta_{0}$ is arbitrary and determines the particular basis states. In this paper we will use $\theta_{0}=-\pi$, so that the angles lie in the range $-\pi \leq \theta_{n}<\pi$. The angle operator $\hat{\phi}_{\theta}$ has as its eigenstates the states $\left|\theta_{n}\right\rangle$ (4) with the associated eigenvalues $\theta_{n}$ (5):

$$
\hat{\phi}_{\theta}=\sum_{n=0}^{2 L} \theta_{n}\left|\theta_{n}\right\rangle\left\langle\theta_{n}\right| .
$$

This operator is, of course, the natural analogue of the phase operator associated with the phase angle for an optical field mode or harmonic oscillator [5]. It is manifestly Hermitian and, by virtue of the fact that it operates on a state space of finite dimension, also self-adjoint. The forms of the angle operator (6) and of the angular momentum operator,

$$
\hat{L}_{z}=\hbar \sum_{m=-L}^{L} m|m\rangle\langle m|,
$$

lead directly to the commutator

$$
\left[\hat{\phi}_{\theta}, \hat{L}_{z}\right]=\frac{2 \pi \hbar}{2 L+1} \sum_{m, m^{\prime} / \text { crcrm } \neq m^{\prime}} \frac{\left(m-m^{\prime}\right) \exp \left[\mathrm{i}\left(m-m^{\prime}\right) \theta_{0}\right]\left|m^{\prime}\right\rangle\langle m|}{\exp \left[\mathrm{i}\left(m-m^{\prime}\right) 2 \pi(2 L+1)\right]-1}
$$

and hence to a uncertainty relation of the Robertson-Schrödinger form [13]

$$
\Delta L_{z} \Delta \phi_{\theta} \geq \frac{1}{2}\left|\left\langle\left[\hat{\phi}_{\theta}, \hat{L}_{z}\right]\right\rangle\right| .
$$

This uncertainty relation is rigorously correct and leads to sensible results. In particular the expectation value of the commutator is zero for both eigenstates of $\hat{L}_{z}$ and $\hat{\phi}_{\theta}$, for which $\Delta L_{z}=0$ and $\Delta \phi_{\theta}=0$ respectively.

Our simpler form of the uncertainty relation (2) arises on applying the limit $L \rightarrow \infty$ to the expectation value of the commutator in (9). If the state has finite moments of $\hat{L}_{z}$ then we can treat $m-m^{\prime}$ in the denominator of (8) as small compared with $2 L+1$ and this leads to the uncertainty relation of the form

$$
\Delta L_{z} \Delta \phi_{\theta} \geq \frac{\hbar}{2}\left|1-(2 L+1)\left\langle\mid \theta_{0}\right\rangle\left\langle\theta_{0} \mid\right\rangle\right| .
$$

In the limit as $L \rightarrow \infty$, our discrete set of angles approaches a continuous limit, with angle density $(2 L+1) / 2 \pi$ and we can introduce a continuous angle wavefunction $\psi(\phi)$ and probability density $P(\phi)=|\psi(\phi)|^{2}$, normalised such that

$$
\int_{\theta_{0}}^{\theta_{0}+2 \pi} P(\phi) \mathrm{d} \phi=1
$$


In this limit our uncertainty relation becomes

$$
\Delta L_{z} \Delta \phi_{\theta} \geq \frac{\hbar}{2}\left|1-2 \pi P\left(\theta_{0}\right)\right|
$$

which, on selecting $\theta_{0}=-\pi$ and using periodicity (which requires $P(-\pi)=P(\pi)$ ), gives (2). We emphasize that (9) is the rigorous result and that (12) is an approximation, which is valid for all physically preparable states. This does not mean, however, that all mathematical operations using (12) and the associated continuous angle variable are allowed. Working directly with the continuous variable admits angular momenta with arbitrarily large values, corresponding to angular Fourier components of arbitrarily high frequency. This introduces the possibility of angular wavefunctions with discontinuous derivatives and means that we need to exercise caution when identifying $\hat{L}_{z}$ with $-\mathrm{i} \hbar(\mathrm{d} / \mathrm{d} \phi)[3]$.

One cautionary example is provided by considering the intelligent states derived in $[3,8,11]$, for which we found the (continuous) angle wavefunction

$$
\psi(\phi)=\frac{1}{N} \exp \left(-\frac{\lambda}{2} \phi^{2}\right), \quad-\pi \leq \phi<\pi .
$$

This wavefunction has angular momentum amplitudes that fall off like $m^{-2}$ for large $m$. This means that the action of $\hat{L}_{z}=-\mathrm{i} \hbar(\mathrm{d} / \mathrm{d} \phi)$ on $\psi(\phi)$ leads to a well behaved, that is square integrable, state, but the application of $\hat{L}_{z}^{2}=-\hbar^{2}\left(\mathrm{~d}^{2} / \mathrm{d} \phi^{2}\right)$ does not. Moreover, we also find a problem with the self-adjointness of $-\mathrm{i} \hbar(\mathrm{d} / \mathrm{d} \phi)$ for this state in that

$$
\int_{-\pi}^{\pi} \psi^{*}(\phi)\left(-\hbar^{2} \frac{\mathrm{d}}{\mathrm{d} \phi^{2}}\right) \psi(\phi) \mathrm{d} \phi \neq \hbar^{2} \int_{-\pi}^{\pi}\left|\frac{\mathrm{d} \psi}{\mathrm{d} \phi}\right|^{2} \mathrm{~d} \phi .
$$

Problems of this kind are, of course, a consequence of performing forbidden mathematical operations (associated with the continuous limit) rather than an indication of problems with the angle or angular momentum operators. Such difficulties can always be resolved, by returning to the $2 L+1$ space and the exact uncertainty relation $(9)$.

\section{Intelligent states}

The equality in the general form of the uncertainty relation [13] leads to a condition for the intelligent states $[8,14]$ :

$$
\left[\hat{L}_{z}-\left\langle\hat{L}_{z}\right\rangle\right]|\psi\rangle=\mathrm{i} \hbar \lambda\left[\hat{\phi}_{\theta}-\left\langle\hat{\phi}_{\theta}\right\rangle\right]|\psi\rangle
$$

where $\lambda$ is a real number. We choose the angle eigenvalues to lie in the range $-\pi$ to $\pi$ so that $\theta_{0}=-\pi$ and, for simplicity of notation, denote the corresponding angle operator $\hat{\phi}_{-\pi}$ as $\hat{\phi}$. It has been shown, moreover, that we can restrict ourselves to cases with zero mean angle and angular momentum $[3,8]$ without loss of generality. The equality condition (15) can be turned into a differential equation for the intelligent states by employing the angle representation $\psi(\phi)$. Solutions for the resulting differential equation

$$
\left[\frac{\partial}{\partial \phi}+\lambda \phi\right] \psi(\phi)=0
$$




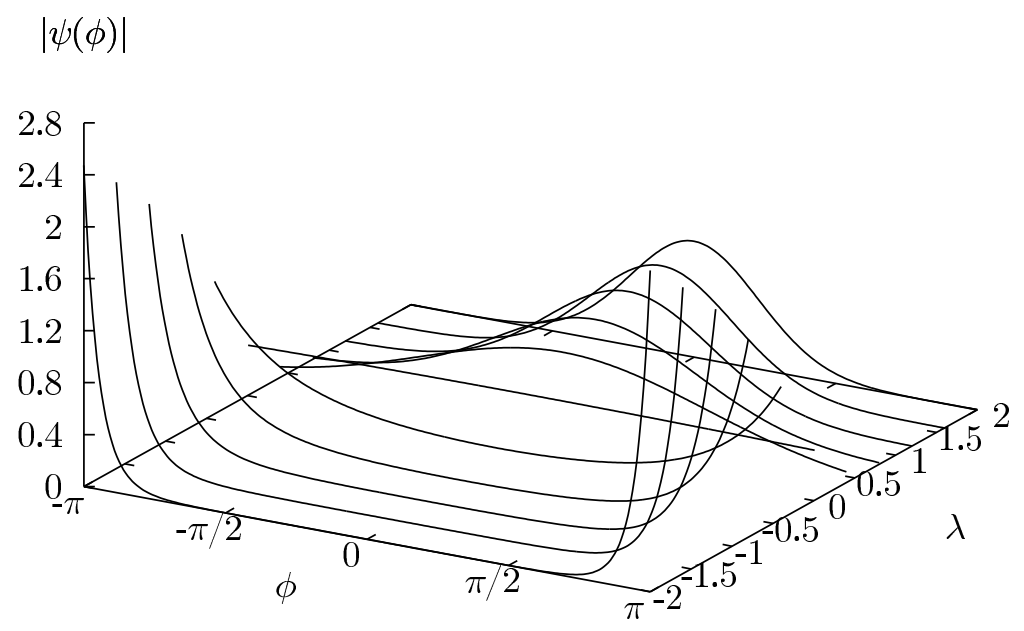

Figure 1. The wavefunction in angle representation for the intelligent states plotted for different values of $\lambda$. The transition from the truncated Gaussians in [8] to the large-uncertainty intelligent states for $\lambda<0$ is shown.

for $\lambda>0$ are the truncated Gaussians (3) [3, 8]. Formally this solution can be extended to negative values of $\lambda$ [11]. For such negative values the wavefunction in the angle representation is given by

$$
\psi(\phi)=\frac{1}{N} \exp \left(-\frac{\lambda}{2} \phi^{2}\right)=\frac{1}{N} \exp \left(\frac{|\lambda|}{2} \phi^{2}\right),
$$

with the normalisation constant

$$
N^{2}=\int_{-\pi}^{\pi} \exp \left(|\lambda| \phi^{2}\right) \mathrm{d} \phi=-\mathrm{i} \sqrt{\frac{\pi}{|\lambda|}} \operatorname{erf}(\mathrm{i} \sqrt{|\lambda|} \pi) .
$$

Note that the complex error function with a purely imaginary argument is also purely imaginary. For $\lambda<0$ the wavefunction grows exponentially and is inversely proportional to the truncated Gaussians (see figure (1)). A state represented by this wavefunction can only exist because it is defined on a finite interval. For the case of linear momentum and position, where Gaussians are both the intelligent and constrained minimum uncertainty product states, solutions can also be characterised by a parameter $\lambda$. However, for the large-uncertainty case with $\lambda<0$ the wavefunctions would not be normalisable and therefore not be elements of the Hilbert space.

\subsection{Angle uncertainty}

The square of the angle uncertainty is given by $(\Delta \phi)^{2}=\left\langle\hat{\phi}^{2}\right\rangle-\langle\hat{\phi}\rangle^{2}$. As our solutions are symmetric about $\phi=0$, the mean value of $\hat{\phi}$ is zero and the angle uncertainty is 
simply the expectation value of $\hat{\phi}^{2}$

$$
(\Delta \phi)^{2}=\int_{-\pi}^{\pi} \phi^{2} P(\phi) \mathrm{d} \phi=\frac{1}{N^{2}} \int_{-\pi}^{\pi} \phi^{2} \exp \left(|\lambda| \phi^{2}\right) \mathrm{d} \phi .
$$

The functional form of the wavefunction allows us to express the angle uncertainty as the derivative of the normalisation constant $N$ with respect to $|\lambda|$ :

$$
(\Delta \phi)^{2}=\frac{1}{N^{2}} \int_{-\pi}^{\pi} \frac{\mathrm{d}}{\mathrm{d}|\lambda|} \exp \left(|\lambda| \phi^{2}\right) \mathrm{d} \phi=\frac{1}{N^{2}} \frac{\mathrm{d} N^{2}}{\mathrm{~d}|\lambda|} .
$$

An expression for the angle uncertainty can be obtained on substituting the analytically exact normalisation constant (18) in (20):

$$
\Delta \phi=\left|\mathrm{i} \sqrt{\frac{\pi}{|\lambda|}} \frac{\exp \left(|\lambda| \pi^{2}\right)}{\operatorname{erf}(\mathrm{i} \sqrt{|\lambda| \pi})}-\frac{1}{2|\lambda|}\right|^{1 / 2} .
$$

The largest angle uncertainty possible for intelligent states is $\Delta \phi=\pi$. This can be seen from (19) as $P(\pi)$ is an even function of $\phi$ for the intelligent states:

$$
(\Delta \phi)^{2}=2 \int_{0}^{\pi} \phi^{2} P(\phi) \mathrm{d} \phi \leq 2 \pi^{2} \int_{0}^{\pi} P(\phi) \mathrm{d} \phi=\pi^{2} .
$$

The maximum value $\Delta \phi=\pi$ is obtained in the limit of $\lambda \rightarrow-\infty$. A plot of $\Delta \phi$ as a function of $\lambda$ is shown in figure 2. For $\lambda=0$ the flat angle probability density $P(\phi)=1 /(2 \pi)$ gives the angle uncertainty of $\Delta \phi=\pi / \sqrt{3}$. The truncated Gaussians with $\lambda>0$ have a smaller angle uncertainty than $\pi / \sqrt{3}$ while the intelligent states with $\lambda<0$ considered here have a larger angle uncertainty.

\subsection{Angular momentum uncertainty}

Using the continuous wavefunction (17) and the representation of $\hat{L}_{z}$ as a derivative requires some care. In section 2 we have argued that for the intelligent states the first derivative of $\psi(\phi)$ with respect to $\phi$ represents a well behaved state, while the second derivative does not. This is the reason why we can use $-\mathrm{i} \hbar(\mathrm{d} / \mathrm{d} \phi)$ as a valid representation of $\hat{L}_{z}$ in the eigenvalue equation for the intelligent states. However, in the arbitrarily large state space of $2 L+1$ dimension reviewed in section 2 the angular momentum operator $\hat{L}_{z}$ is self-adjoint and Hermitian, and we may therefore write for the expectation value of $\hat{L}_{z}^{2}$ for the intelligent state $|\psi\rangle$ :

$$
\left\langle\psi \mid \hat{L}_{z}^{2} \psi\right\rangle=\left\langle\hat{L}_{z} \psi \mid \hat{L}_{z} \psi\right\rangle=\|\left|\hat{L}_{z} \psi\right\rangle \|^{2}
$$

where $\|\cdot\|$ symbolises the norm of a state vector. The expectation value is a physical quantity and we can make now the transition to the continuous wavefunction $\psi(\phi)=\langle\phi \mid \psi\rangle$. Following the argument given above it is then valid to replace $\hat{L}_{z}$ with $-\mathrm{i} \hbar(\mathrm{d} / \mathrm{d} \phi)$, allowing $(\Delta m)^{2}$ to be written as

$$
(\Delta m)^{2}=\frac{1}{\hbar^{2}}\left\langle\hat{L}_{z}^{2}\right\rangle=\frac{1}{N^{2}} \int_{-\pi}^{\pi}\left|\frac{\mathrm{d}}{\mathrm{d} \phi} \psi(\phi)\right|^{2} \mathrm{~d} \phi .
$$




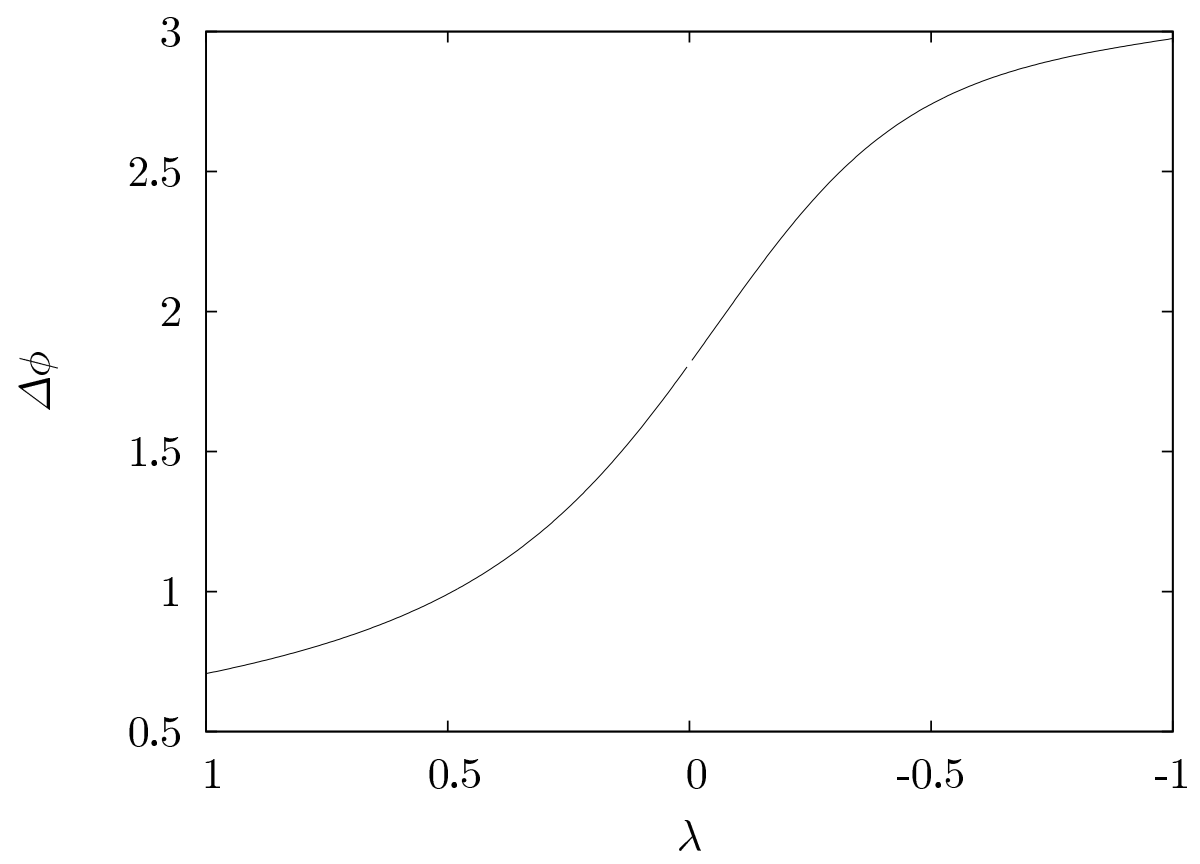

Figure 2. The angle uncertainty $\Delta \phi$ for the intelligent states. The parameter value $\lambda=0$ distinguishes between the truncated Gaussians with $\lambda>0[8]$ and the largeuncertainty intelligent states with $\lambda<0$. For intelligent states the angle uncertainty is bounded by $\Delta \phi \leq \pi$.

On substituting the wavefunction (17) into this expression we find that the angular momentum uncertainty $\Delta m$ can be expressed in terms of the angle uncertainty $\Delta \phi$ :

$$
(\Delta m)^{2}=\frac{1}{N^{2}} \int_{-\pi}^{\pi}|\lambda|^{2} \phi^{2} \exp \left(|\lambda| \phi^{2}\right) \mathrm{d} \phi .=|\lambda|^{2}(\Delta \phi)^{2} .
$$

If we use the expression for the angle uncertainty $\Delta \phi$ from (21) we obtain for the angular momentum uncertainty $\Delta m$ :

$$
\Delta m=|\lambda|\left|\mathrm{i} \sqrt{\frac{\pi}{|\lambda|}} \frac{\exp \left(|\lambda| \pi^{2}\right)}{\operatorname{erf}(\mathrm{i} \sqrt{|\lambda| \pi})}-\frac{1}{2|\lambda|}\right|^{1 / 2} .
$$

To obtain the equality in the uncertainty relation for linear position and momentum, the uncertainties have to be inversely proportional to each other. In the angular case the uncertainties are directly proportional to each other; if we have a large angle uncertainty we require also a large angular momentum uncertainty to satisfy the equality. The reason for this behaviour lies in the state dependent right hand side of (2). A plot of the angular momentum uncertainty for positive and negative values of $\lambda$ is shown in figure (3). For $\lambda=0$ we have the flat angle probability distribution and an angular momentum eigenstate $|m\rangle$ with zero angular momentum uncertainty $\Delta m=0$. In our case, with zero angular momentum mean, we have $m=0$ and the eigenstate $|0\rangle$. 


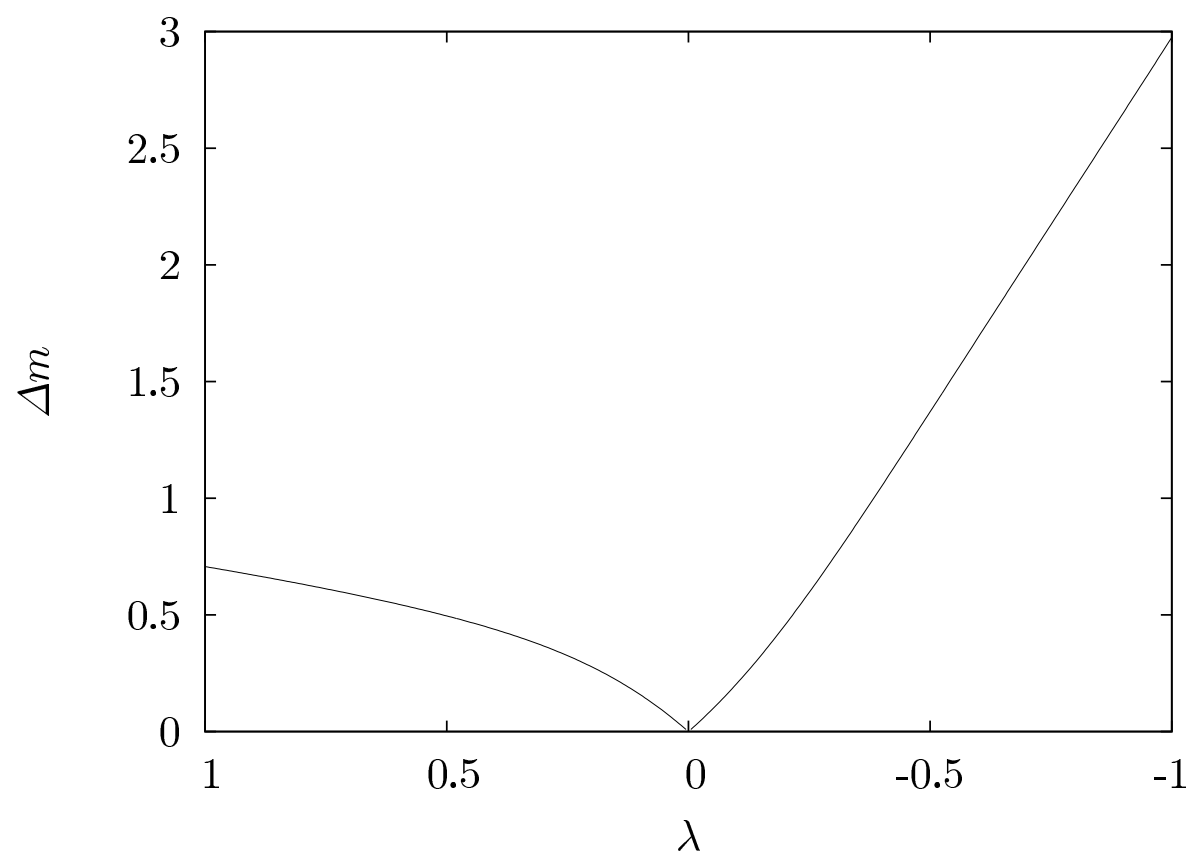

Figure 3. The angular momentum uncertainty $\Delta m$ plotted as a function of $\lambda$. From (25), which also holds for $\lambda>0$, one can see that $\Delta m$ tends to infinity for $\lambda \rightarrow \infty$. For $\lambda \rightarrow-\infty, \Delta m$ tends also to infinity and for this case the behaviour is visible in the plot.

\subsection{Uncertainty product}

The left hand side of the uncertainty relation (2) is given by the uncertainty product $\Delta m \Delta \phi$. Using the result for the angular momentum uncertainty $\Delta m$ (25) and the expression for $\Delta \phi(21)$ the uncertainty product can be expressed as

$$
\Delta m \Delta \phi=|\lambda|(\Delta \phi)^{2}=\mathrm{i} \sqrt{\pi|\lambda|} \frac{\exp \left(|\lambda| \pi^{2}\right)}{\operatorname{erf}(\mathrm{i} \sqrt{|\lambda| \pi})}-\frac{1}{2}
$$

The right hand side of the uncertainty relation is given by $|1-2 \pi P(\pi)| / 2$. For the large-uncertainty intelligent states with $\lambda<0, P(\pi)$ is always larger than $1 /(2 \pi)$. The modulus can thus be replaced by $2 \pi P(\pi)-1$. Using the wavefunction (17) and the normalisation (18) to express $P(\pi)$ yields the equality with the uncertainty product. The behaviour of the uncertainty product as a function of $\Delta \phi$ is shown in figure 4 . For $\Delta \phi<\pi / \sqrt{3}$ this is the same plot as in [8]. From figure 4 and equation (27) it is clear that for the intelligent states with $\lambda<0$ the uncertainty product can become arbitrarily large. 


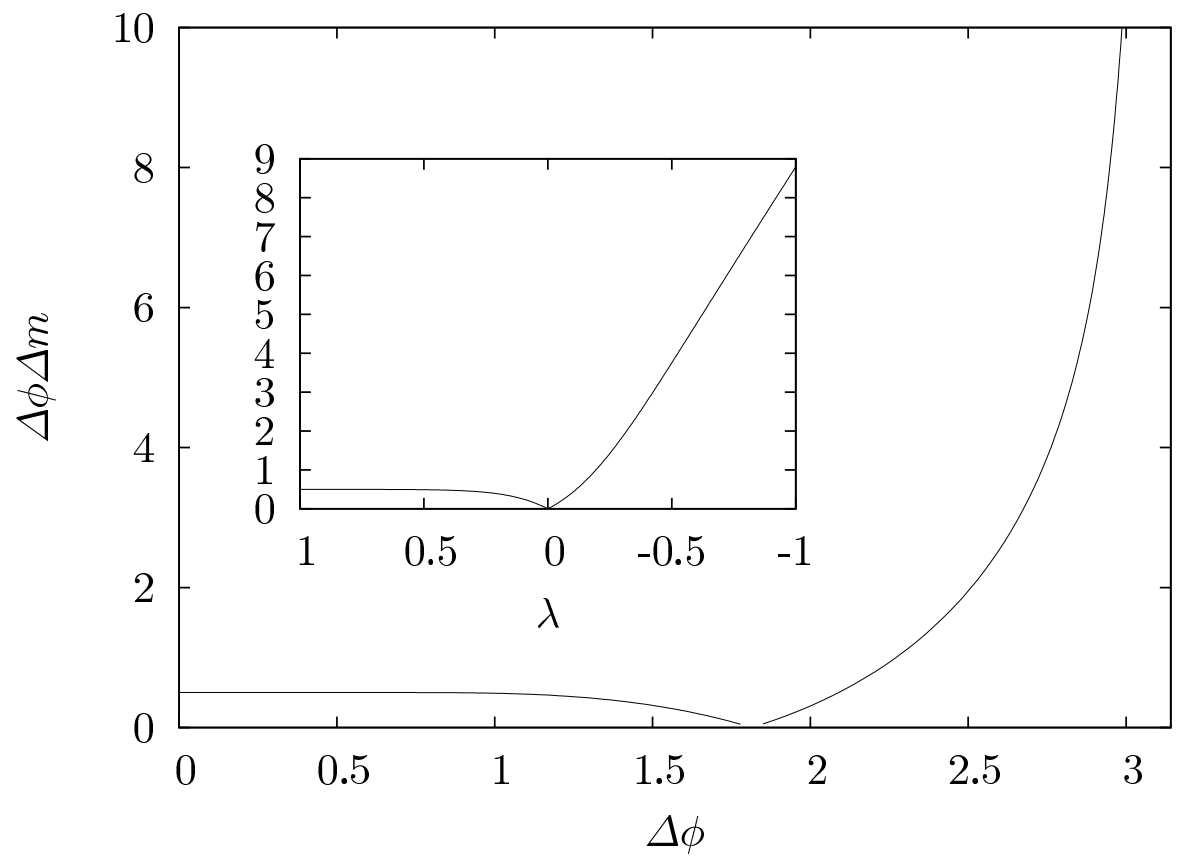

Figure 4. Plot of the uncertainty product $\Delta m \Delta \phi$ against $\Delta \phi$. For the largeuncertainty intelligent states $(\Delta \phi>\pi / \sqrt{3}$ or $\lambda<0)$ the uncertainty product has no upper bound. The inset shows a plot of the uncertainty product against $\lambda$ for comparison with the plots of $\Delta \phi$ and $\Delta m$ (see figures 2 and 3; see also a similar plot in [11])

\subsection{Angular momentum distribution}

The probability amplitudes of the orbital angular momentum $c_{m}$ are calculated from the wavefunction (17) by means of a Fourier transform:

$$
c_{m}=\frac{1}{\sqrt{2 \pi}} \int_{-\pi}^{\pi} \psi(\phi) \exp (-\mathrm{i} m \phi) \mathrm{d} \phi .
$$

By virtue of the symmetry of the wave function the transformation may be specialised to the Fourier cosine transform. The result can be expressed in terms of a complex error function

$$
c_{m}=\frac{(-1)^{m}}{N \sqrt{2 \pi}} \exp \left(\frac{m^{2}}{2|\lambda|}\right) \sqrt{\frac{\pi}{2|\lambda|}} 2 \Im\left[\operatorname{erf}\left(\sqrt{\frac{1}{2|\lambda|}} m+\mathrm{i} \sqrt{\frac{|\lambda|}{2}} \pi\right)\right],
$$

where $\Im$ denotes the imaginary part of a complex number. The divergent behaviour of the positive exponent is counterbalanced by the imaginary part of the complex error function, which decays quickly to zero in this case. This results in an angular momentum distribution which is similar to a Lorentzian (see figure (5)). 

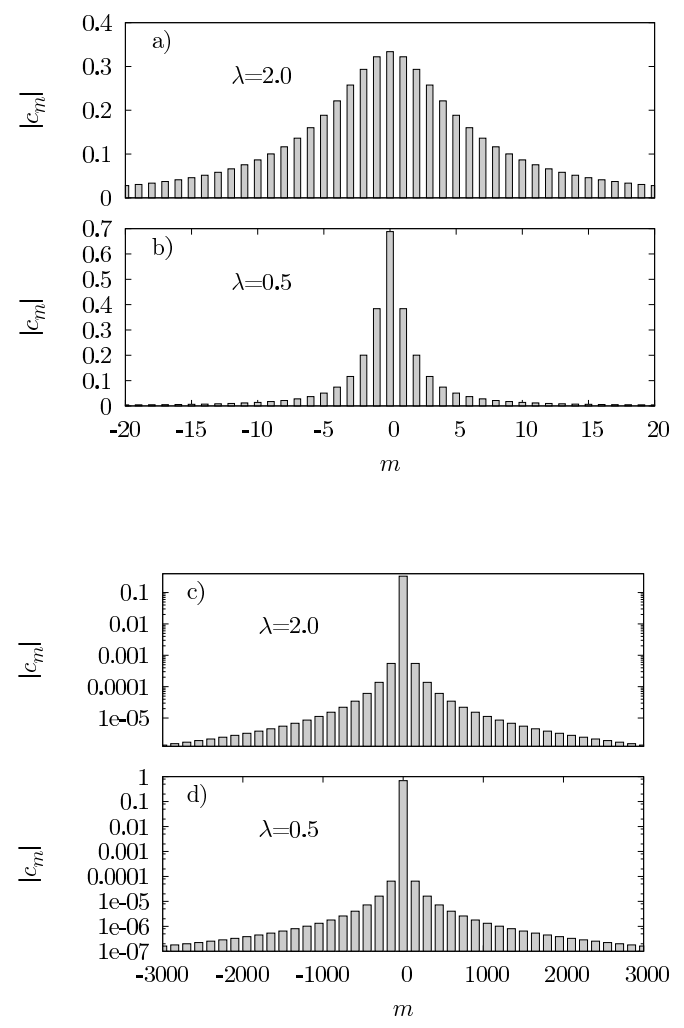

Figure 5. Orbital angular momentum distribution for different values of $\lambda$. a) $\lambda=-2.0$ : A linear plot showing the distribution for the central $m$ values. b) $\lambda=-0.5$ : linear plot. c) $\lambda=-2.0$ : A logarithmic plot for the flanks. The width of the bars in the plot covers $100 \mathrm{~m}$ values, but the shown value corresponds to the $m$ value in the middle of the bar. d) $\lambda=-0.5$ : logarithmic plot.

\section{Limiting behaviour}

In the following we investigate the large-uncertainty intelligent states for two limiting cases to develop an understanding for the behaviour of the angle and angular momentum uncertainties. For small $|\lambda|$ we present a perturbative approach, while for large $\lambda$ we approximate the exponential in the wavefunction (17).

\subsection{Small $|\lambda|$ approximation}

For small values of $|\lambda|$ the continuous angle probability density becomes a flat function of the angle and in the limit of $\lambda \rightarrow 0$, where $P(\phi)=1 /(2 \pi)$ we have an angular momentum eigenstate. As we consider the case with zero angular momentum mean $\left\langle\hat{L}_{z}\right\rangle=0$, the angular momentum eigenstate in the limit of $\lambda \rightarrow 0$ is $|0\rangle$. The behaviour of $\Delta \phi$ in this parameter region can be explained using a perturbation ansatz for $|0\rangle$ with $\lambda$ as perturbation parameter. As explained in section 2 we start the derivation of $\Delta \phi$ in this perturbative approach within an arbitrarily large, but finite state space of 
$2 L+1$ dimension. For zero angle and angular momentum mean the condition for the intelligent state (15) can be written as

$$
\hat{L}_{z}|\psi\rangle-\mathrm{i} \lambda \hat{\phi}|\psi\rangle=0 \text {. }
$$

For small $\lambda$ we can use the perturbation ansatz $|\psi\rangle=|0\rangle+\lambda\left|\varphi^{1}\right\rangle$. Substituting this ansatz in the condition (30) yields at first order in $\lambda$

$$
\lambda\left(\hat{L}_{z}\left|\varphi^{1}\right\rangle-\mathrm{i} \hat{\phi}|0\rangle\right)=0 .
$$

Without loss of generality we can write the perturbative state $|\varphi\rangle$ as a superposition of angular momentum eigenstates $|m\rangle, m=-L,-L+1, \ldots,-1,1, \ldots, L$ without a contribution from $|0\rangle$ :

$$
\left|\varphi^{1}\right\rangle=\sum_{m=-L / c r c r m \neq 0}^{L} c_{m}^{1}|m\rangle .
$$

The angular momentum eigenstate $|0\rangle$ is a physical state, that is a state which may be approximated to any desired accuracy by the expansion $\sum_{m} b_{m}|m\rangle$, where the coefficients $b_{m}=0$ for $|m|>M$. Here, the sum includes all integer values of $m$ and the bound $M$ is sufficiently large to guarantee the desired accuracy but always less than $L$. Restricting the domain of the angle operator to these physical states simplifies the expression for the angle operator and changes the summation to include an infinite number of angular momentum states $|m\rangle$. Using the definitions for the angular momentum operator and for the angle operator for physical states in [7] we can calculate the resulting states $\hat{L}_{z}\left|\varphi^{1}\right\rangle$ and $\hat{\phi}|0\rangle$ :

$$
\begin{aligned}
& \hat{L}_{z}\left|\varphi^{1}\right\rangle=\sum_{m=-L / c r c r m \neq 0}^{L} m c_{m}^{1}|m\rangle=\sum_{m=-L}^{L} m c_{m}^{1}|m\rangle, \\
& \hat{\phi}|0\rangle=-\mathrm{i} \sum_{m \neq 0} \frac{\exp (\mathrm{i} m \pi)}{m}|m\rangle .
\end{aligned}
$$

On substituting these results in the condition for the intelligent states at first order in $\lambda(31)$ we find an equation to determine the $c_{m}^{1}$ :

$$
\lambda \sum_{m \neq 0}\left(m c_{m}^{1}-\frac{\exp (\mathrm{i} m \pi)}{m}\right)|m\rangle=0 .
$$

For the linearly independent basis sates $|m\rangle$ the coefficients in (31) have to vanish to give zero as result. This requires $c_{m}^{1}=-(-1)^{m} / m^{2}$. For small $\lambda$ the perturbative state $|\psi\rangle$ may thus be written as

$$
|\psi\rangle=\frac{1}{N_{\text {per }}}\left(|0\rangle-\lambda \sum_{m \neq 0} \frac{(-1)^{m}}{m^{2}}|m\rangle\right),
$$

where $N_{\text {per }}$ is the normalisation constant for the perturbative wavefunction. The angular momentum amplitudes of the state $|\psi\rangle$ are given by $c_{0}=1 / N_{\text {per }}$ and $c_{m}=\lambda c_{m}^{1} / N_{\text {per }}$. The angle representation of this state is obtained by projection on an angle eigenstate. We hereby calculate a physical quantity, the angle probability amplitude, and we can now 
allow $L$ to tend to infinity, which yields the continuous wavefunction of the perturbative state:

$$
\begin{aligned}
\langle\phi \mid \psi\rangle=\psi(\phi) & =\frac{1}{N_{\text {per }}} \frac{1}{\sqrt{2 \pi}}\left(1-\lambda \sum_{m \neq 0} \frac{(-1)^{m}}{m^{2}} \exp (\mathrm{i} m \phi)\right) \\
& =\frac{1}{N_{\text {per }}} \frac{1}{\sqrt{2 \pi}}\left[1+\lambda\left(\frac{\pi^{2}}{6}-\frac{\phi^{2}}{2}\right)\right] .
\end{aligned}
$$

The sum in the last equation has been evaluated using the contour integration method [15], which can also be used to calculate the normalisation from the requirement that the angular momentum probabilities $\left|c_{m}\right|^{2}$ sum to unity:

$$
\sum_{m}\left|c_{m}\right|^{2}=1=\left(1+\lambda^{2} \sum_{m \neq 0} \frac{1}{m^{4}}\right)=\left(1+\lambda^{2} \frac{\pi^{4}}{45}\right) \frac{1}{N_{\text {per }}^{2}} .
$$

The square of the uncertainty for the perturbative state can be calculated at first order from the continuous wavefunction (37):

$$
(\Delta \phi)^{2}=\left\langle\hat{\phi}^{2}\right\rangle=\frac{1}{N_{\text {per }}^{2}} \frac{1}{2 \pi} \int_{-\pi}^{\pi} \phi^{2}\left[1+\lambda\left(\frac{\pi^{2}}{6}-\frac{\phi^{2}}{2}\right)\right] \mathrm{d} \phi .
$$

At first order in $\lambda$ this leads to the angle uncertainty

$$
\Delta \phi \approx \frac{\pi}{\sqrt{3}}\left(1-\frac{2}{15} \lambda \pi^{2}\right)
$$

which explains the behaviour of $\Delta \phi$ for small $\lambda$ (see figure 6). The angular momentum uncertainty can be derived from the angle uncertainty according to (25). Because the angle uncertainty is multiplied by $|\lambda|$ and the perturbative $\Delta \phi$ from (40) is at first order $\lambda$ this approximation holds for the second order in $\lambda$

$$
\Delta m \approx|\lambda| \frac{\pi}{\sqrt{3}}\left(1-\frac{2}{15} \lambda \pi^{2}\right)
$$

The behaviour of the angular momentum uncertainty in the perturbative approach is shown in figure 7 . The equality in the uncertainty relation (2) at first order in $\lambda$ can be seen directly from the results for $\Delta \phi(40)$ and $\Delta m(41)$. The uncertainty product is given by

$$
\Delta m \Delta \phi=|\lambda| \frac{\pi^{2}}{3}+\mathrm{O}\left(|\lambda|^{2}\right)
$$

After substituting the expression for $N_{\text {per }}$ from (38) into the wavefunction (37), the right hand side of the uncertainty relation becomes

$$
\frac{1}{2}|1-2 \pi P(\pi)| \frac{1}{2}\left|1-\left(1-2|\lambda| \frac{\pi^{2}}{3}+\mathrm{O}\left(|\lambda|^{2}\right)\right)\right|=|\lambda| \frac{\pi^{2}}{3}+\mathrm{O}\left(|\lambda|^{2}\right)
$$

which shows the equality in the uncertainty relation to the first order in $|\lambda|$. The uncertainty product as a function of $\Delta \phi$ is shown in figure 8 . 


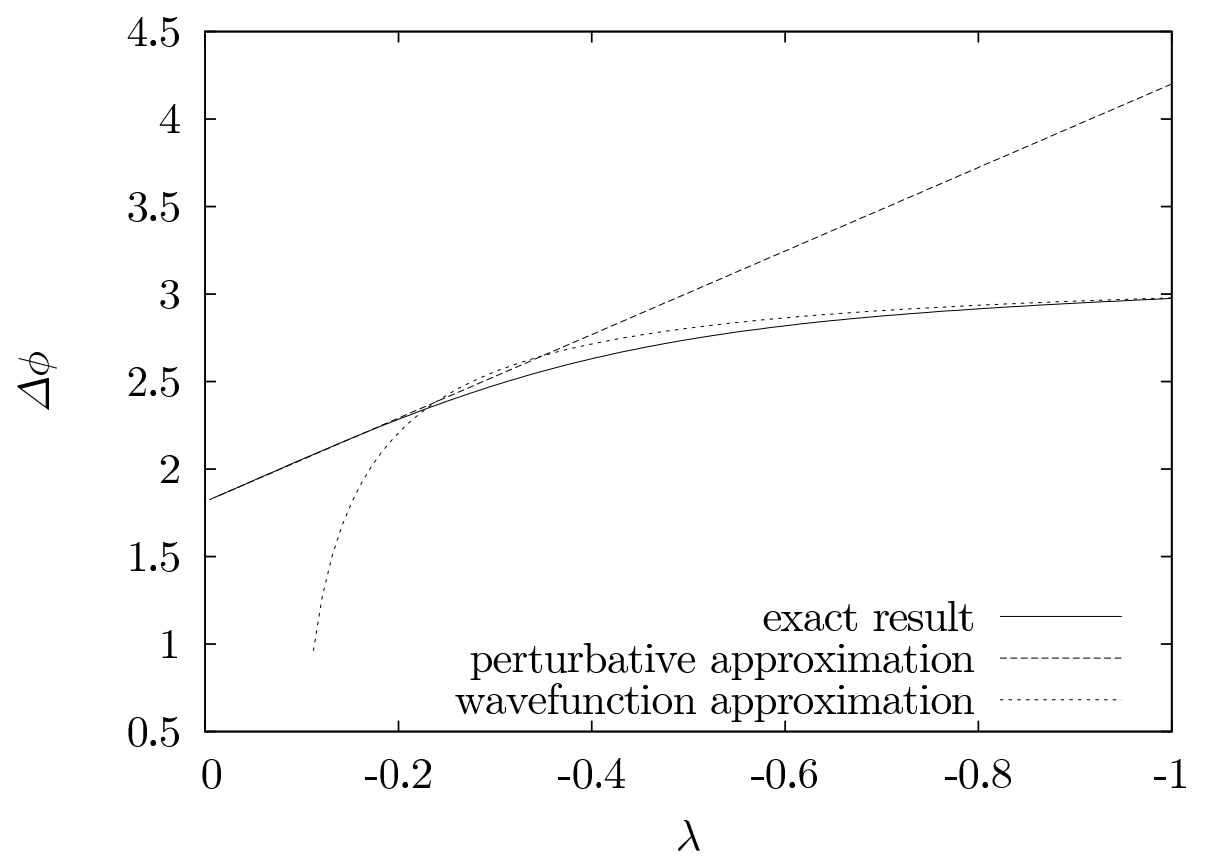

Figure 6. The angle uncertainty $\Delta \phi$ plotted for the analytically exact expression (21), in the perturbative approximation and in the wavefunction approximation (47). The wavefunction approximation fails for small values of $|\lambda|$. In this region a perturbative approximation can explain the behaviour of $\Delta \phi$.

\subsection{Large $|\lambda|$ approximation}

In the approximation for large $|\lambda|$ we approximate the wavefunction within an integration. This leads to two variants of this approximation: approximating the wavefunction in the normalisation integral gives a simple elementary expression, which can be used to calculate the angle and angular momentum uncertainties and hence the uncertainty product. We will refer to this approach as wavefunction approximation. On the other hand, applying this approximation under a Fourier integral to calculate the angular momentum probability amplitudes, allows us to explain the angular momentum distribution and to calculate the angular momentum uncertainty in this approximation. This approach will be called Lorentzian approximation as it explains the Lorentzian shape of the angular momentum distribution.

4.2.1. Wavefunction approximation The interesting quantities for the uncertainty relation are all related to the normalisation constant: $\Delta m=|\lambda| \Delta \phi$ by virtue of (25) and $\Delta \phi$ is related to the normalisation according to (20). An approximation for the normalisation constant in the limit of large $|\lambda|$ provides simple approximate expressions, which explain the behaviour of the uncertainties in this limit. 


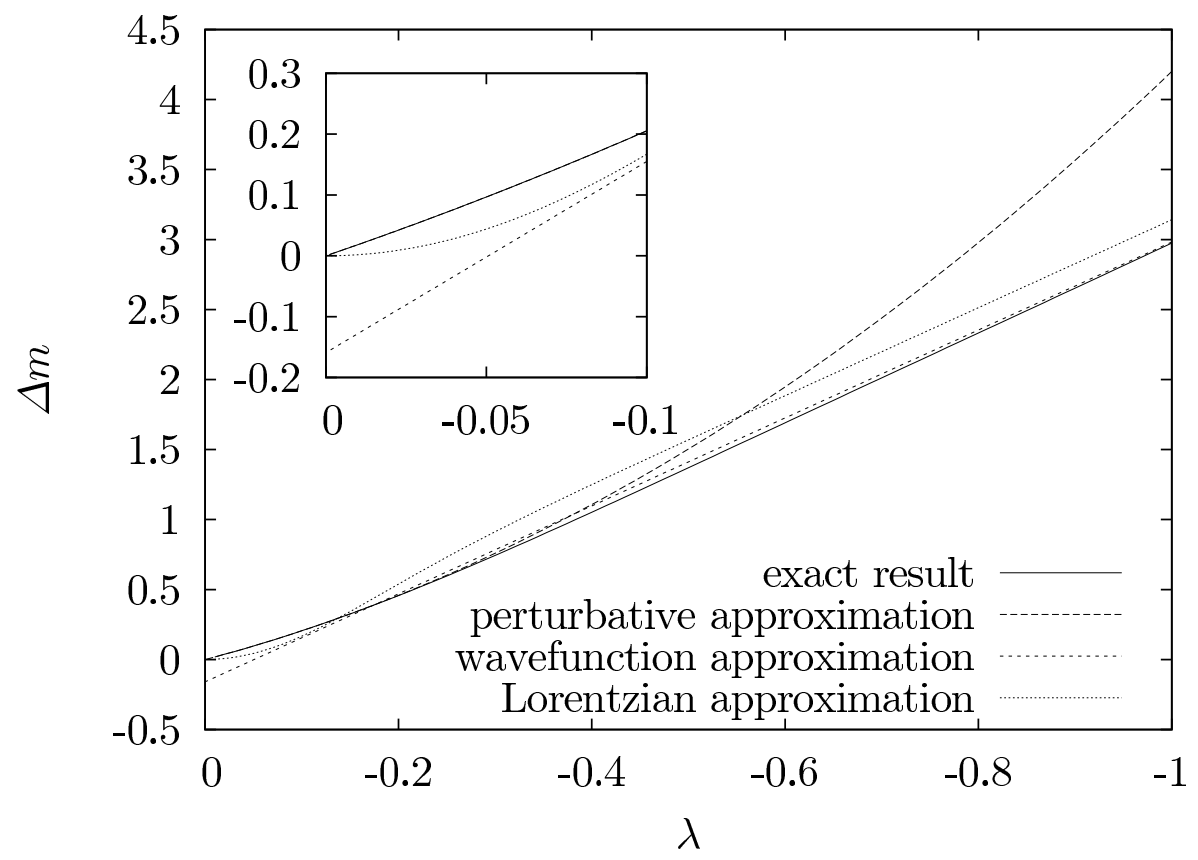

Figure 7. Plot of the angular momentum uncertainty for the exact result and different approximations. The expressions for $\Delta m$ in the wavefunction and perturbative approximation is derived from (25) by substituting the corresponding expression for $\Delta \phi$. For very small $|\lambda|$ the perturbative approximation is indistinguishable from the exact result. The Lorentzian approximation originates from a summation of the Lorentzian angular momentum probabilities in (57).

The normalisation integral (18) can be rewritten as

$$
N^{2}=2 \exp \left(|\lambda| \pi^{2}\right) \int_{-\pi}^{0} \exp [|\lambda|(\phi+\pi)(\phi-\pi)] \mathrm{d} \phi .
$$

For a large $|\lambda|$ only a small region around $-\pi$ will contribute significantly to the integral, and we can therefore approximate the factor $(\phi-\pi)$ in the exponential with $-2 \pi$ and extend the upper integration boundary to infinity. This results in the normalisation constant $N_{\text {wvf }}$

$$
N_{\mathrm{wvf}}^{2}=2 \exp \left(|\lambda| \pi^{2}\right) \int_{-\pi}^{\infty} \exp (-2 \pi|\lambda|(\phi+\pi)) \mathrm{d} \phi=\frac{1}{|\lambda| \pi} \exp \left(|\lambda| \pi^{2}\right) .
$$

Using this normalisation constant (45) in the expression for the probability density yields $P(\pi)$ this approximation. From (17) we have:

$$
P(\pi) \approx|\lambda| \pi \text { for }|\lambda| \pi^{2}>3 .
$$

We thus can give an approximation of the right hand side of the uncertainty relation (2) in the limit of large $|\lambda|$. The angle and angular momentum uncertainties in the left 
hand side can be obtained using (20) and (25). Substituting $N_{\text {wvf }}$ into (20) yields a simple expression for the angle uncertainty:

$$
\Delta \phi \approx \frac{|\lambda| \pi}{\exp \left(|\lambda| \pi^{2}\right)} \frac{\mathrm{d}}{\mathrm{d}|\lambda|} \frac{\exp \left(|\lambda| \pi^{2}\right)}{|\lambda| \pi}=\pi^{2}-\frac{1}{|\lambda|} .
$$

A graph of the angle uncertainty is given in figure 6 . One can see that the wavefunction approximation gives a good agreement with the analytical values for $|\lambda|>1 / 3$ but fails for smaller values, where $1 /|\lambda|$ grows without bound. The angular momentum uncertainty in this approximation follows from substituting $\Delta \phi(47)$ in (25)

$$
\Delta m \approx|\lambda| \pi \sqrt{1-\frac{1}{|\lambda| \pi^{2}}} \approx|\lambda| \pi-\frac{1}{2 \pi} .
$$

This approximate expression for $\Delta m$ gives a good approximation for large $|\lambda|$ as can be seen in figure 7.

In the approximation for large values of $|\lambda|$ the uncertainty product $\Delta m \Delta \phi$ is given as a simple function of $|\lambda|$. From (47) and (48) follows:

$$
\Delta m \Delta \phi \approx|\lambda| \pi^{2}-1 \text {. }
$$

The right hand side of the uncertainty relation (2) contains the probability density $P(\pi)$ which is approximately $|\lambda| \pi$ from (46) for $|\lambda| \pi^{2} \mid>3$. Therefore, $2 \pi P(\pi)=2|\lambda| \pi^{2}>1$, and the equality in the uncertainty relation is expressed by

$$
|\lambda| \pi^{2}-1 \simeq|\lambda| \pi^{2}-\frac{1}{2} \text { for }|\lambda| \pi^{2}>3 .
$$

Clearly, for sufficiently large values of $|\lambda|$ the discrepancy becomes negligible. The approximation for large $\lambda$ is based on our expression for $N_{\text {wvf }}$. If we refine the approximation in (45) by setting $\exp [|\lambda|(\phi+\pi)(\phi-\pi)]=\exp [-2 \pi|\lambda|(\phi+\pi)] \exp [|\lambda|(\phi-$ $\left.\pi)^{2}\right]$ and expanding the second exponential according to $\exp \left[|\lambda|(\phi-\pi)^{2}\right] \approx 1+|\lambda|(\phi-\pi)^{2}$, we find that the expression for $P(\pi)$ changes to

$$
P(\pi)=\lambda \pi\left(1-\frac{1}{2} \frac{1}{\pi^{2}|\lambda|}\right)^{-1} .
$$

Substituting this expression in the right hand side of the uncertainty relation (2) gives the equality with the uncertainty product (49). A plot of the uncertainty product is given in figure 8. Compared to the good agreement with the numerical values for the separate uncertainties $\Delta \phi$ and $\Delta m$, the discrepancies for the product appear to be large for the wavefunction approximation in figure 8. This is because $\Delta m=\lambda \Delta \phi$ [cf. equation (25)] and as we have plotted the uncertainty product against $\Delta \phi$, the effect of an error in our approximation for $\Delta \phi$ is enhanced in this plot.

\subsection{Lorentzian approximation}

The exact angular momentum distribution has a shape similar to a Lorentzian (see figure 5). We therefore seek to approximate the Fourier kernel as an exponentially decaying 


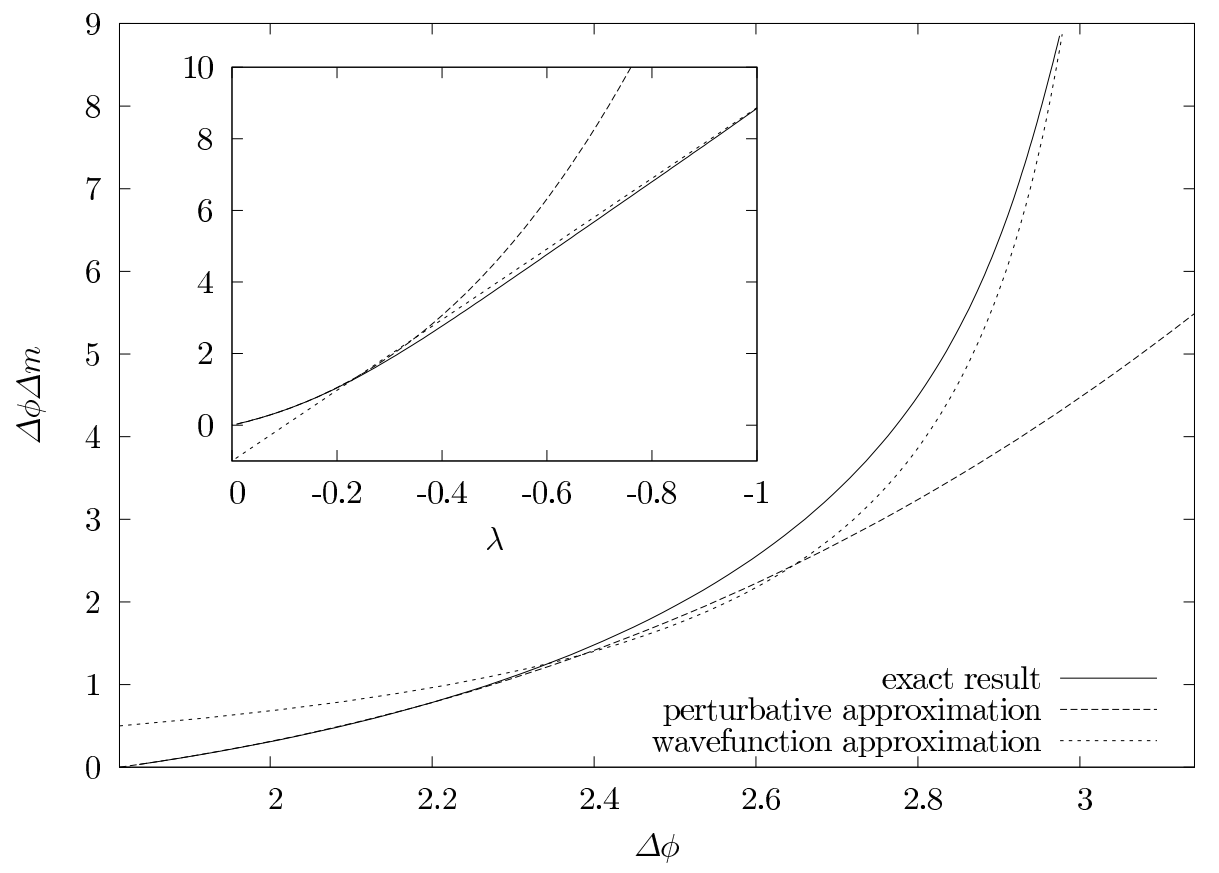

Figure 8. Plot of the uncertainty product $\Delta m \Delta \phi$ as a function of $\Delta \phi$. For negative $\lambda \Delta \phi$ ranges between $\pi / \sqrt{3}$ and $\pi$. The curves are for the kernel wavefunction approximation for large $|\lambda|$ and for the perturbative approximation for small $|\lambda|$. The inset shows the uncertainty product plotted as a function of $\lambda$.

function. Owing to the symmetry of the wavefunction the Fourier cosine transform can be used:

$$
c_{m}=\frac{2}{N \sqrt{2 \pi}} \int_{-\pi}^{0} \exp \left(\frac{|\lambda|}{2} \phi^{2}\right) \cos (m \phi) \mathrm{d} \phi .
$$

In essence the integrand can be approximated in the same way as for the wavefunction approximation. The Fourier integrand can be written as $\exp [|\lambda|(\phi+\pi)(\phi-$ $\pi) / 2] \exp \left(|\lambda| \pi^{2} / 2\right)$. Within the integration interval $-\pi \leq \phi<0$ and for large $|\lambda|$ this Fourier kernel is only significantly different from zero around the lower boundary $-\pi$. This allows us to approximate the kernel with $\exp [|\lambda|(\phi+\pi)(-\pi)] \exp \left(|\lambda| \pi^{2} / 2\right)$ and to extend the integration interval in (52) to infinity, which results in an angular momentum distribution in the shape of a Lorentzian:

$$
\begin{aligned}
c_{m} & \approx \frac{1}{N \sqrt{2 \pi}} \exp \left(\frac{|\lambda|}{2} \pi^{2}\right) \int_{-\pi}^{\infty} \exp (-\pi|\lambda|(\phi+\pi)) \cos (m \phi) \mathrm{d} \phi, \\
& =\frac{1}{N \sqrt{2 \pi}}(-1)^{m} \exp \left(\frac{|\lambda|}{2} \pi^{2}\right)\left(\frac{2|\lambda| \pi}{|\lambda|^{2} \pi^{2}+m^{2}}\right) .
\end{aligned}
$$

The Lorentzian and wavefunction approximation are very similar but not identical. This is because extending the upper integration boundary is not an equally good 

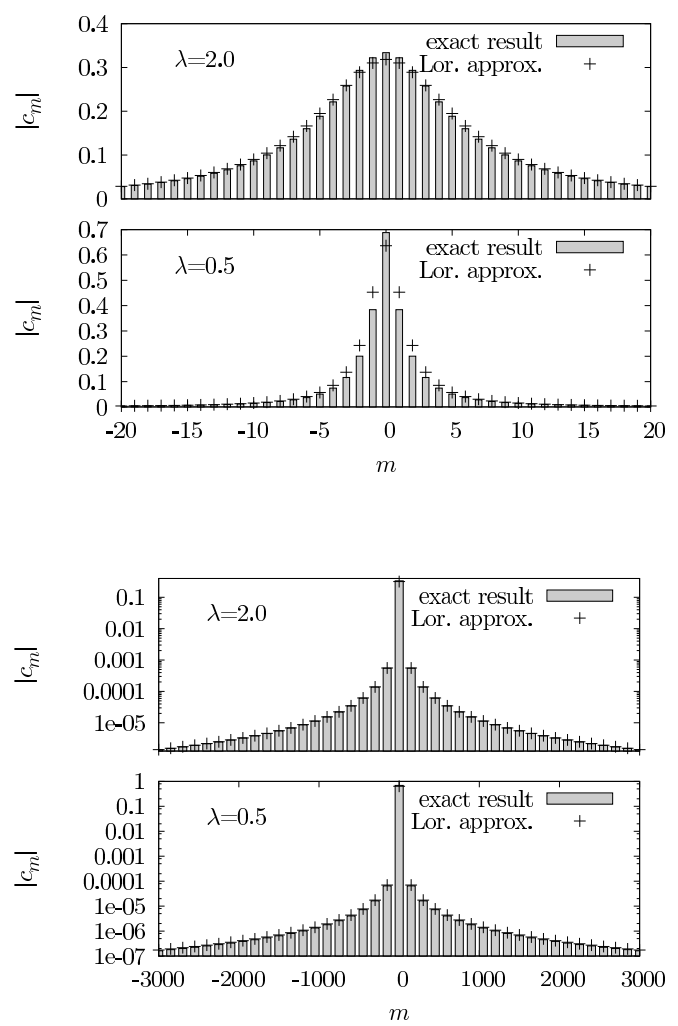

Figure 9. Orbital angular momentum distribution for different values of $\lambda$. a) $\lambda=-2.0$ : linear plot shows the distribution for the central $m$ values. b) $\lambda=-0.5$ : linear plot. c) $\lambda=-2.0$ : logarithmic plot for the flanks. The width of the bars in the plot covers $100 m$ values, but the shown value corresponds to the $m$ value in the middle of the bar. d) $\lambda=-0.5$ : logarithmic plot.

approximation for the two integrals in (53) and (45). One can see from figure (9) that the agreement with the Lorentzian is better at the flanks and for higher values of $\lambda$. This is consistent with our considerations as for larger values of $|\lambda|$ the justification for the approximation of the wavefunction in the Fourier integral becomes more valid. Also, for central values of $m$ the region around the upper boundary in the Fourier integral (52) contributes more. The angular momentum probabilities have to sum to unity. This condition can be used to calculate the normalisation constant within the Lorentzian approximation. We denote this approximate normalisation constant with $N_{\text {Lor }}$ to distinguish it from the analytically exact value $N$. Using the results for the probability amplitudes $c_{m}$ in (53) the sum of the probabilities may be written as:

$$
\sum_{m=-\infty}^{\infty}\left|c_{m}\right|^{2}=1=\frac{1}{N_{\text {Lor }}^{2} 2 \pi} 4|\lambda|^{2} \pi^{2} \exp \left(|\lambda| \pi^{2}\right) \sum_{m=-\infty}^{\infty}\left(|\lambda|^{2} \pi^{2}+m^{2}\right)^{-2} .
$$


The summation can be executed using contour integration [15], which results in the following expression for $N_{\text {Lor }}$ :

$$
N_{\text {Lor }}^{2}=\exp \left(|\lambda| \pi^{2}\right)\left(\pi \operatorname{cosech}^{2}\left(|\lambda| \pi^{2}\right)+\frac{1}{|\lambda| \pi} \operatorname{coth}\left(|\lambda| \pi^{2}\right)\right) .
$$

The angular momentum uncertainty can also be calculated from the angular momentum probabilities $\left|c_{m}\right|^{2}$ in the Lorentzian approximation (53) in the same way as the normalisation constant $N_{\text {Lor }}$. From figure (9) and equation (53) it can be seen that the angular momentum mean is zero. The square of the uncertainty is thus given by:

$$
(\Delta m)^{2} \approx \frac{1}{N_{\text {Lor }}^{2} 2 \pi} 4|\lambda|^{2} \pi^{2} \exp \left(|\lambda| \pi^{2}\right) \sum_{m=-\infty}^{\infty} \frac{m^{2}}{\left(|\lambda|^{2} \pi^{2}+m^{2}\right)^{2}} .
$$

Using again contour integration to evaluate the sum [15] and substituting the value of $N_{\text {Lor }}$ we can write for the angular momentum uncertainty in the Lorentzian approximation:

$$
\begin{aligned}
(\Delta m)^{2} & =\sum_{m}\left|c_{m}\right|^{2} m^{2} \\
& \approx \pi^{2}|\lambda|^{2} \frac{\operatorname{coth}\left(|\lambda| \pi^{2}\right)-|\lambda| \pi^{2} \operatorname{cosech}^{2}\left(|\lambda| \pi^{2}\right)}{\operatorname{coth}\left(|\lambda| \pi^{2}\right)+|\lambda| \pi^{2} \operatorname{cosech}^{2}\left(|\lambda| \pi^{2}\right)} .
\end{aligned}
$$

For $|\lambda| \pi^{2}>3$ this expression simplifies because the hyperbolic function $\operatorname{cosech}\left(|\lambda| \pi^{2}\right)$ becomes negligible small. In this limit we have

$$
(\Delta m) \approx \pi|\lambda| .
$$

The behaviour of the angular momentum uncertainty as a function of $\lambda$ can be seen in figure (7).

\section{Conclusion}

We have studied the uncertainty relation for angular momentum and angle in a series of recent papers. The progress in creating and manipulating optical states with orbital angular momentum made it possible to confirm the form of the angular uncertainty relation experimentally for intelligent states, that is states which obey the equality in the uncertainty relation [8]. As the angular uncertainty relation has a state dependent lower bound, the intelligent states do not necessarily minimize the uncertainty product. We have explored the difference between the intelligent states and states, which give a minimum in the uncertainty product under additional constraints in a second paper [3]. In the present paper we emphasize the difference between intelligent states and minimum product states by introducing a class of intelligent states with arbitrarily large uncertainty product.

The difference between the two kinds of intelligent states is given by the sign of a parameter $\lambda$. For positive $\lambda$ we have the truncated Gaussians with an uncertainty product bounded by $\hbar / 2$. For negative $\lambda$ the wavefunction grows exponentially towards the edge of the chosen radian range, resulting in an arbitrarily large uncertainty 
product. We have derived analytical expressions for the angular momentum and angle uncertainties and the uncertainty product. In two limiting cases, for small negative $\lambda$ and large negative $\lambda$ we have explained the behaviour of the uncertainties with the parameter $\lambda$ using approximate results. The three papers combined give an extensive overview of the uncertainty relation for angular momentum and angle.

\section{Acknowledgments}

We would like to thank David T Pegg for helpful discussions and we acknowledge financial support from the UK Engineering and Physical Sciences Research Council (EPSRC) under the grant GR S03898/01 and the Royal Society of Edinburgh.

\section{References}

[1] Heisenberg W 1927 Z. Phys. 43127

[2] Aragone C, Guerri G, Salamó S and Tani J L 1974 J. Phys. A: Math. Gen. 7 L149 Aragone C, Chalbaud E and Salamó S 1976 J. Math. Phys. 171963

[3] Pegg D T, Barnett S M, Zambrini R, Franke-Arnold S and Padgett M 2005 New J. Phys. 762

[4] Jackiw R 1968 J. Math. Phys 9339

[5] Barnett S M and Pegg D T 1989 J. Mod. Opt 367 Pegg D T and Barnett S M 1989 Phys. Rev. A 391665

Pegg D T and Barnett S M 1997 J. Mod. Opt 44225

[6] Barnett S M and Radmore P 1997 Methods in Theoretical Quantum Optics (Oxford: Clarendon Press)

[7] Barnett S M and Pegg D T 1990 Phys. Rev. A 413427

[8] Franke-Arnold S, Barnett S M, Yao E, Leach J, Courtial J and Padgett M 2004 New J. Phys. 6 103

[9] Allen L, Beijersbergen M W, Spreeuw R J C and Woerdman P 1992 Phys. Rev A 458185

Allen L, Barnett S M and Padgett M 2003 Optical Angular Momentum (Bristol: Institute of Physics Publishing)

[10] Vaziri A, Weihs G and Zeilinger A 2002 J. Opt. B: Quantum Semiclass. Opt. 4 S47

Leach J, Padgett M J, Barnett S M, Franke-Arnold S and Courtial J 2002, Phys. Rev. Lett. 88 257901

[11] Galindo A and Pascual P 1990 Quantum Mechanics vol 1 (Berlin: Spinger Verlag)

[12] Judge D and Lewis J T 1963 Phys. Rev. Lett. 5190

Carruthers P and Nieto M M 1968 Rev. Mod. Phys. 40441

[13] Robertson H P 1929 Phys. Rev 34163

Schrödinger E 1930 Sitz. Preuss. Akad. Wiss. XIX 296

[14] Schwabl F 2002 Quantum Mechanics 3rd edn (Berlin: Springer Verlag)

[15] Stephenson G and Radmore P M 1993 Advanced Mathematical Methods for Engineering and Science Students (Cambridge: Cambridge University Press) 